\title{
Racial Disparity in 30-Day Outcomes of Metabolic and Bariatric Surgery
}

\author{
Haleh Amirian ${ }^{1} \cdot$ Alfonso Torquati $^{2} \cdot$ Philip Omotosho $^{2}$ \\ Published online: 19 November 2019 \\ (C) Springer Science+Business Media, LLC, part of Springer Nature 2019
}

\begin{abstract}
Background There is evidence of racial disparity in the long-term weight loss outcomes of bariatric surgery. However, there has been a more limited evaluation of the impact of race on immediate perioperative outcomes. The aim of this study was to compare 30-day postoperative outcomes among different races.

Study Design The 2016 Metabolic and Bariatric Surgery Accreditation and Quality Improvement Program (MBSAQIP) database was queried to identify patients aged $\geq 18$ and body mass index $\geq 35$ who underwent primary laparoscopic Roux-en-Y gastric bypass (LRYGB) or laparoscopic sleeve gastrectomy (LSG) with known information on race. We then evaluated the effect of five different races on four different 30-day outcomes.

Results Of the total 106,932 patients (79.5\% White, 19.3\% African American (AA), 0.5\% Asian, 0.4\% American Indian or Alaska Native, $0.3 \%$ Native Hawaiian or other Pacific Islander), 30-day rates of postoperative complication, readmission, reintervention, and reoperation were $6,3.8,1.3$, and 1.2\%, respectively. After controlling for other covariates in multivariate logistic regression and selecting White as reference, AA was the only race associated with a higher risk of postoperative complications (odds ratio [OR] 1.13; confidence interval [CI] 1.06-1.2) and readmissions (OR 1.47; CI 1.3-1.6). AA and American Indian or Alaska Native were also associated with higher re-interventions (OR 1.31; CI 1.15-1.51 and OR 2.11; CI 1.03-4.34). Furthermore, AA was associated with lower 30-day reoperations (OR 0.83; CI 0.7-0.9).

Conclusion This study found significant racial differences in short-term outcomes following bariatric surgery. Factors underlying these disparities are unclear and warrant further investigation.
\end{abstract}

Keywords Racial disparities · Perioperative outcomes

\section{Introduction}

Obesity is a chronic disease with a globally rising rate that reached $30.1 \%$ in 2017 [1]. Metabolic and bariatric surgery has been proved to be the most effective method not only for weight loss [2, 3] but also for resolving or improving associated comorbidities [4-7] and quality of life as well as longterm mortality in obese patients [8].

The abstract has been submitted for ACS Clinical Congress 2019.

Philip Omotosho

Philip_omotosho@rush.edu

Graduate College, Rush University, Chicago, IL, USA

2 Division of Minimally Invasive and Bariatric Surgery, Rush University Medical Center, 1653 W. Congress Pkwy, Jelke Building, Suite 604, Chicago, IL 60612, USA
Although the obesity rate has had an upward trend in almost all racial groups, some races are disproportionately affected. Based on the Centers for Disease Control (CDC) reports, Asian adults had the lowest obesity prevalence in 2017 while non-Hispanic black adults (39\%) had the highest, followed by American Indian/Alaska Native (38.7\%), Hispanic (32.4\%), and non-Hispanic White adults $(29.3 \%)$ [1]. Among proposed etiologies for this disproportionate distribution are socio-economic factors and their effects on nutrition [9], genetic differences [10], and possible ethnic differences in fat metabolism [11]. Additionally, there is evidence of gender disparity with female dominance [12] and unequal utilization of bariatric surgery with fewer African Americans undergoing these procedures compared with Whites [13] despite a higher obesity rate in the former. Furthermore, there is evidence of disparate long-term response to bariatric procedures especially disproportionate success in weight loss among different races [14-16]. 
While there are numerous studies on these aspects of disparity in metabolic and bariatric surgery especially related to longterm outcomes, there has been limited evaluation of the impact of race on the short-term postoperative outcomes of these procedures, which constitutes the primary aim of this study.

\section{Methods}

\section{Data Source}

Data were obtained from the 2016 Metabolic and Bariatric Surgery Accreditation and Quality Improvement Program (MBSAQIP) participant use files (PUF) including cases with operation dates between January 1, 2016, and December 31, 2016. With over 790 participating sites across the USA and Canada, the MBSAQIP data registry is the largest bariatricspecific dataset containing preoperative, intraoperative, and postoperative data for patients undergoing metabolic and bariatric surgery. All data are collected by trained and certified metabolic and bariatric surgical clinical reviewers (MBSCRs), and selected participating centers are regularly audited to ensure the data collected are of the highest quality [17].

\section{Study Population}

This study included patients with age $\geq 18$ years and $\mathrm{BMI} \geq 35$ who underwent either laparoscopic Roux-en-Y gastric bypass (LRYGB) or laparoscopic sleeve gastrectomy (LSG), the two most commonly performed bariatric procedures. We used the current procedural terminology (CPT) for the principal operative procedure selection: LRYGB $(43644,43645)$, LSG (43775). We excluded those with unknown race or ethnicity information, previous bariatric surgery, revisional or conversional cases, emergency cases, and any surgical approach other than conventional laparoscopic approach.

\section{Outcomes}

The outcomes of interest were 30-day postoperative outcomes including composite "postoperative complication" defined as presence of any of the 24 adverse events: superficial incisional surgical site infection (SSI), deep incisional SSI, organ/space SSI, wound disruption, sepsis, septic shock, urinary tract infection (UTI), acute renal failure (ARF), progressive renal insufficiency, mechanical ventilation $>48 \mathrm{~h}$, unplanned intubation, pneumonia, unplanned admission to ICU, deep venous thrombosis (DVT), pulmonary embolism (PE), cardiac arrest, myocardial infarction (MI), stroke, coma $>24 \mathrm{~h}$, peripheral nerve injury, Clostridium difficile colitis, dehydration requiring treatment, incisional hernia, and presence of operative drain at 30 days. Other 30-day postoperative outcomes considered as endpoints were at least one readmission, re-intervention, and reoperation.

\section{Statistical Analysis}

Five different races (White, Black or African American, Asian, American Indian or Alaska Native, Native Hawaiian or other Pacific Islander) were compared for basic characteristics and comorbidities, perioperative factors, and postoperative outcomes using chi-square or Fisher's exact tests for categorical variables and analysis of variance (ANOVA) for continuous variables. Multivariate logistic regression models were used to examine the effect of race on outcomes and included patient characteristics, comorbidities, and perioperative factors with $p<0.05$ found in univariate analyses for each outcome. Odds ratios (OR) along with $95 \%$ confidence intervals (CI) were reported, and inference was based on a twosided 5\% level. The analysis was performed using SPSS (Version 22, Chicago, IL).

\section{Results}

The study cohort consisted of 106,932 patients who underwent LRYGB or LSG. The majority were White (79.5\%) followed by Black or African American (AA) (19.3\%), Asian (0.5\%), American Indian or Alaska Native $(0.4 \%)$, and Native Hawaiian or other Pacific Islanders $(0.3 \%)$. Table 1 demonstrates the baseline characteristics in these five groups, most of which are different in at least two of the racial groups. The majority of patients were females who underwent LSG, with a significant gap between the two procedures, except in Native Hawaiian or other Pacific Islander, in which case, the gap became closer. AA and Native Hawaiian or other Pacific Islander patients had significantly higher BMI in comparison with Whites. Additionally, AA patients tended to smoke less within 1 year of surgery when compared with Whites, while American Indian or Alaska Natives had the highest percentage of smokers (13\%) among others. There were also significantly more patients with American Society of Anesthesiologists (ASA) class $>2$ among AAs and Native Hawaiian or other Pacific Islanders when compared with Whites.

Tables 2, 3, and 4 highlight the comorbidities, peri- and postoperative outcomes between racial groups. All comparisons are made with White patients as the reference. AAs had higher rates of hypertension, inferior vena cava (IVC) filter in anticipation of the surgery, renal insufficiency, and requiring or being on dialysis, while lower rates of most other comorbidities. Asian patients had higher rates of diabetes $(33.5 \%)$ and obstructive sleep apnea $(44.4 \%)$. American Indian or Alaska 


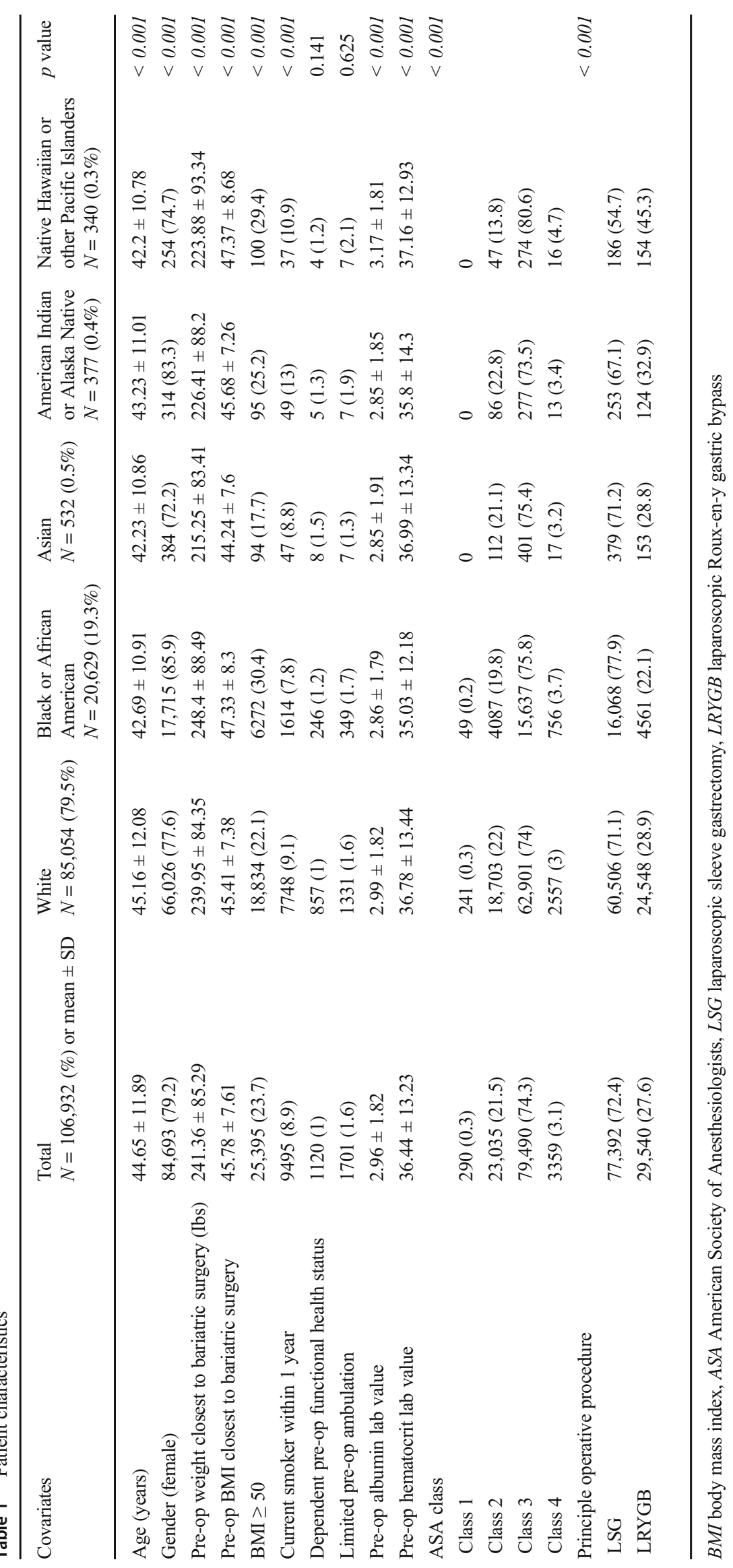




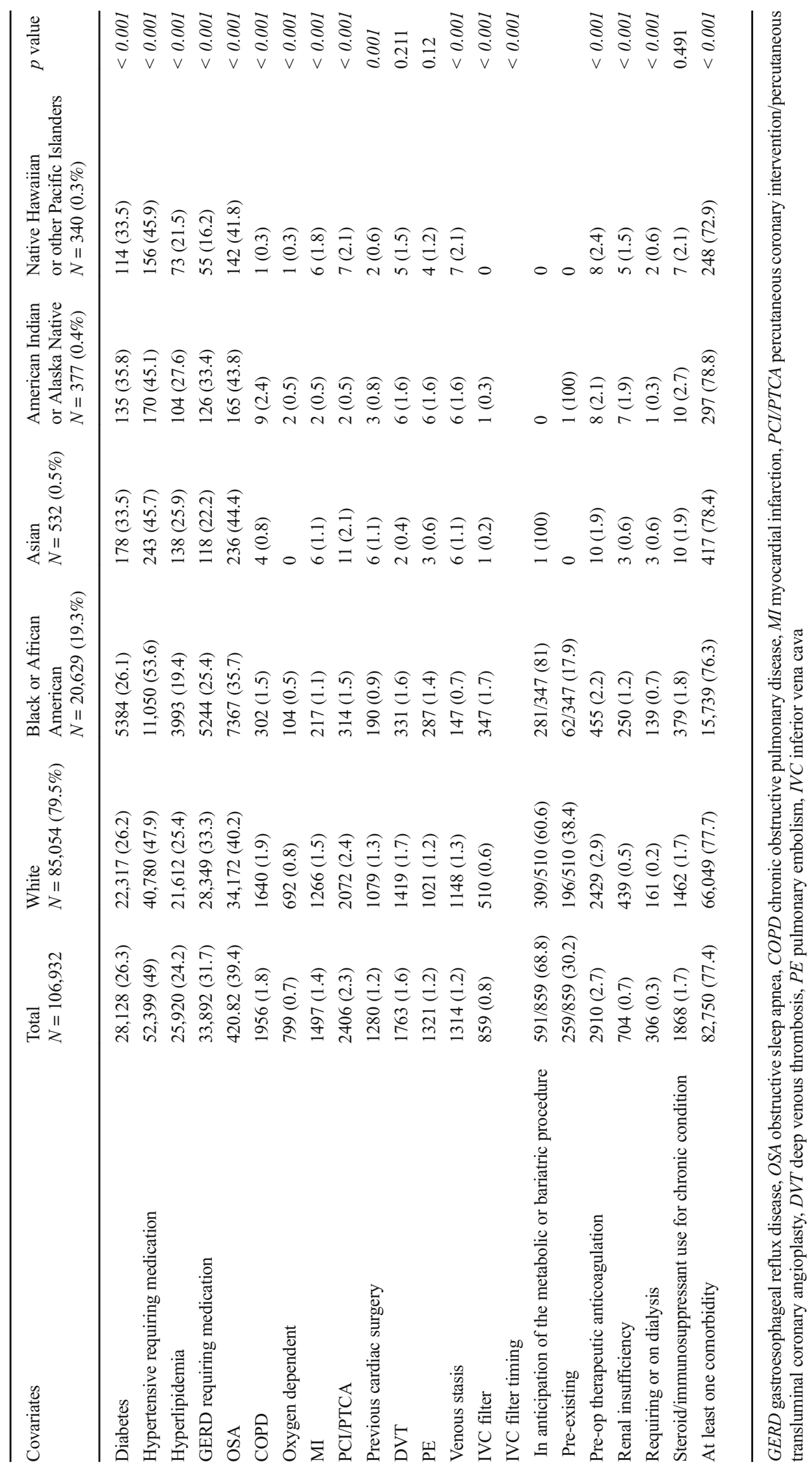




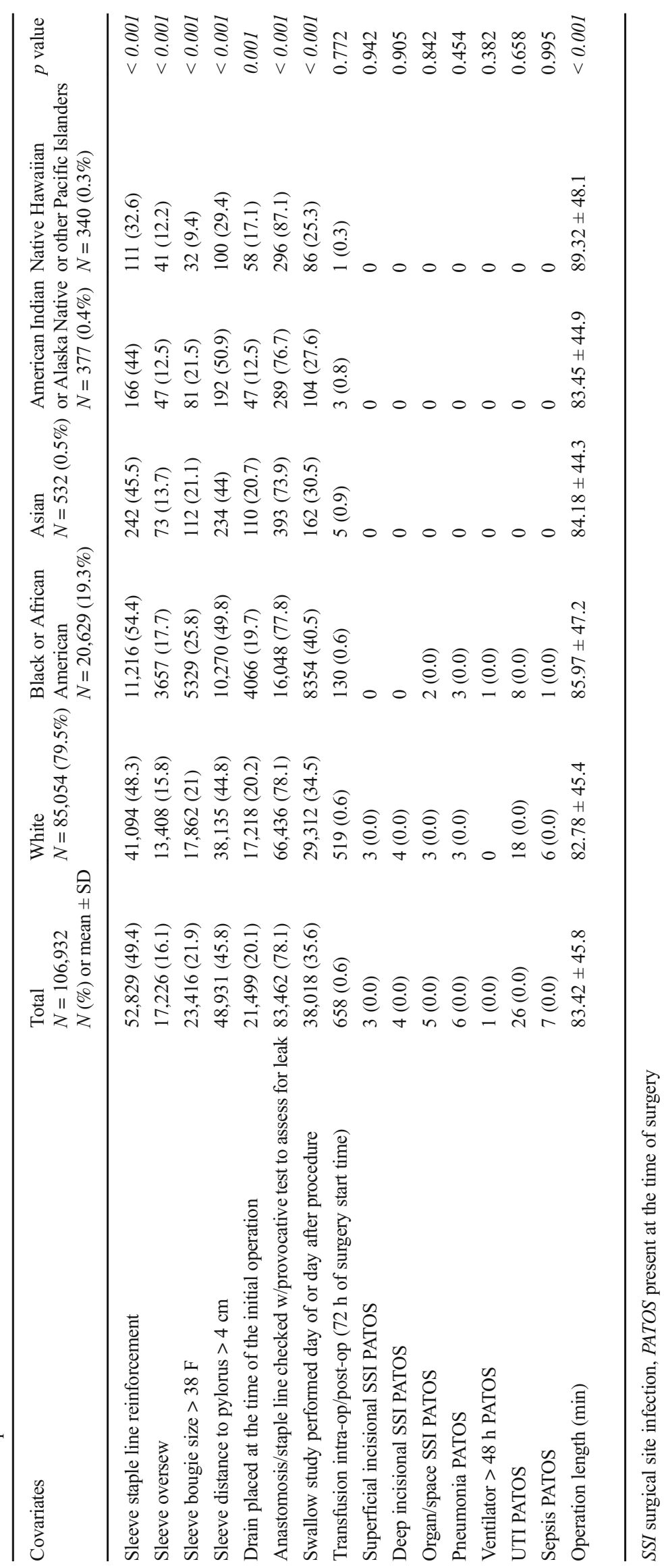




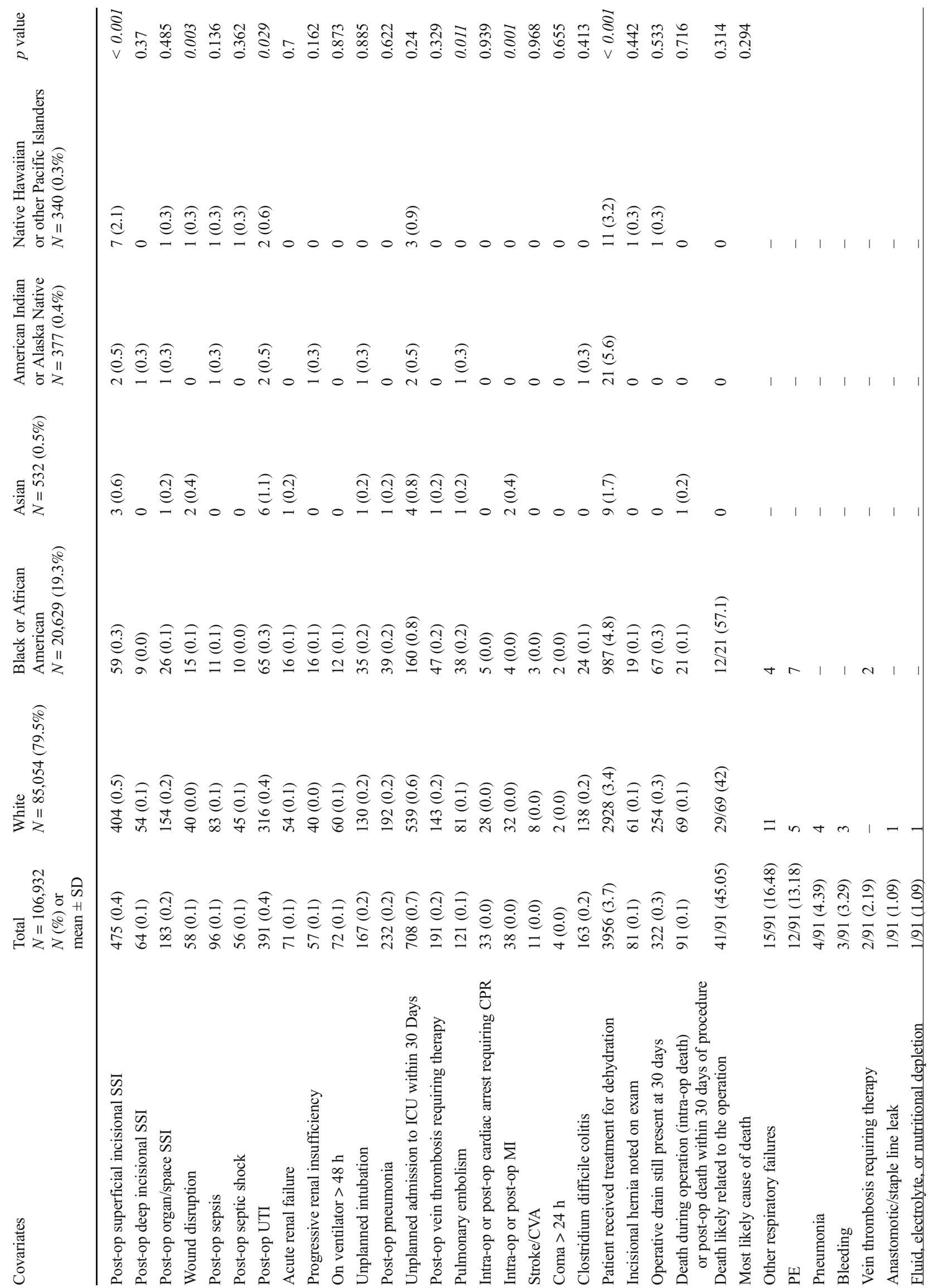




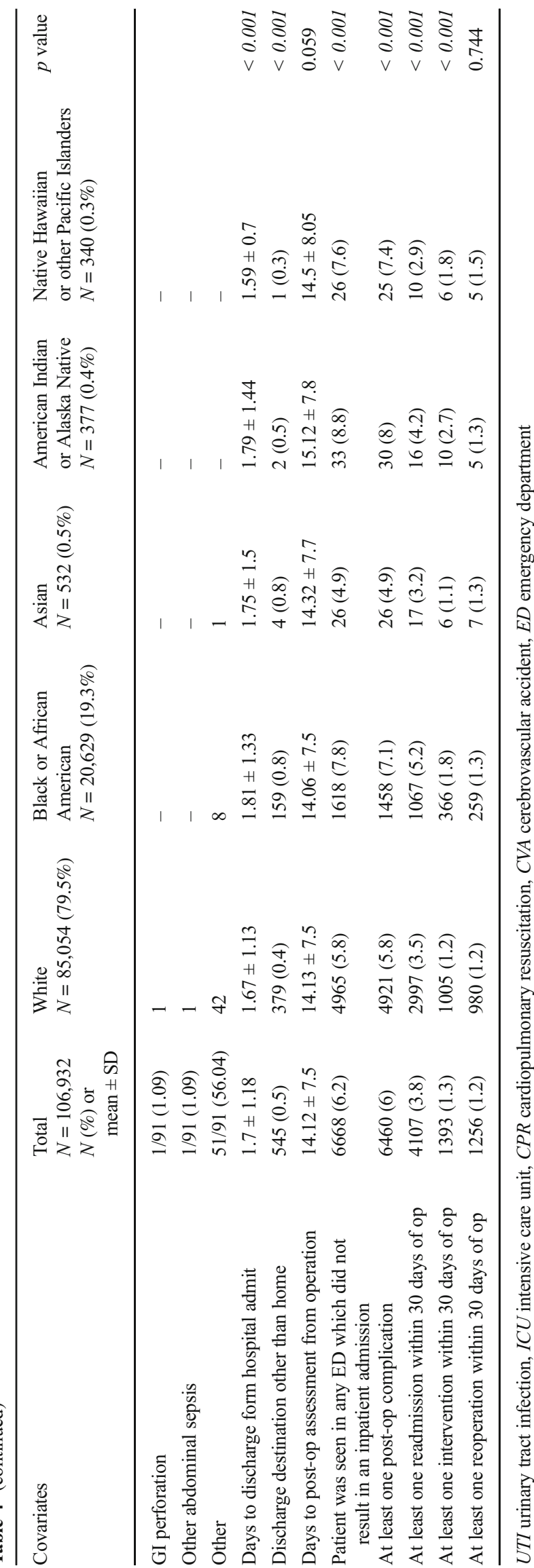

Native and Native Hawaiian or other Pacific Islander also had higher rates of diabetes and renal insufficiency (Table 2). Regarding the perioperative factors, there were some technical differences among racial groups as well as difference in operation length, which was significantly higher in AAs (Table 3).

With regards to postoperative complications, AAs had significantly higher rates of $\mathrm{PE}$ and also longer length of stay, while Asian patients had higher wound disruption, UTI, and MI. Native Hawaiian or other Pacific Islanders, on the other hand, had similar postoperative outcomes to White patients except higher superficial incisional SSI (Table 4).

Although the reoperation rate was not significantly different between groups in the beginning, it was significantly lower in the AA group $(\mathrm{OR}=0.831,95 \% \mathrm{CI}$ 0.7-0.9) after adjusting for other factors. AA was also the only group, which significantly increased the odds of any postoperative complication $(\mathrm{OR}=1.134,95 \% \mathrm{CI}$ $1.064-1.209)$ and readmission $(\mathrm{OR}=1.477,95 \% \mathrm{CI}$ 1.358-1.606). For the 30-day postoperative intervention, American Indian or Alaska Native as well as AA were the two groups that increased the odds of this event in the adjusted analyses (Table 5).

\section{Discussion}

We demonstrated racial disparity in four short-term postoperative outcomes after LRYGB and LSG using the largest available bariatric-specific database. Race was an independent risk factor for 30-day postoperative complications, readmission, and re-intervention, and African Americans had higher odds for all of these outcomes with exception of reoperation, in which they had a lower odds compared with Whites. In addition, re-intervention was significantly higher in American Indian or Alaska Natives when Whites considered as the reference group.

In this study, re-interventions were related to the metabolic and bariatric procedure in $88 \%$ of cases. Nausea and vomiting, fluid, electrolyte, or nutritional depletion was the most common etiology in all races except in Asians. Additionally, diagnostic and therapeutic endoscopies were most common types of intervention performed in all racial groups. Similar to reintervention, majority of reoperations were related to the bariatric procedure $(77 \%)$. Bleeding was the primary reason, and although reoperation type significantly varied among different races, re-exploration (not otherwise qualified) was the most common type in all groups.

Sheka and colleagues [18] have recently performed a similar study on MBSAQIP 2015. However, they only compared Black and White groups. They further classified the study population based on the principal procedure (LRYGB, LSG) 
Table 5 Racial disparity in four 30-day postoperative outcomes

\begin{tabular}{|c|c|c|c|}
\hline 30-day perioperative outcomes & $\begin{array}{l}\text { Adjusted } p \\
\text { value }\end{array}$ & $\begin{array}{l}\text { Odds ratio } \\
(\mathrm{OR})\end{array}$ & $\begin{array}{l}95 \% \text { confidence interval } \\
\text { (CI) }\end{array}$ \\
\hline \multicolumn{4}{|l|}{ Postoperative complications } \\
\hline Black or African American & $<0.001$ & 1.134 & $1.064-1.209$ \\
\hline Asian & 0.236 & 0.781 & $0.519-1.176$ \\
\hline American Indian or Alaska Native & 0.201 & 1.286 & $0.874-1.893$ \\
\hline $\begin{array}{l}\text { Native Hawaiian or other Pacific } \\
\text { Islanders }\end{array}$ & 0.211 & 1.301 & $0.861-1.966$ \\
\hline \multicolumn{4}{|l|}{ Readmission } \\
\hline Black or African American & $<0.001$ & 1.477 & $1.358-1.606$ \\
\hline Asian & 0.904 & 0.967 & $0.562-1.666$ \\
\hline American Indian or Alaska Native & 0.654 & 0.87 & $0.474-1.597$ \\
\hline $\begin{array}{l}\text { Native Hawaiian or other Pacific } \\
\text { Islanders }\end{array}$ & 0.12 & 0.539 & $0.247-1.175$ \\
\hline \multicolumn{4}{|l|}{ Re-intervention } \\
\hline Black or African American & $<0.001$ & 1.319 & $1.151-1.512$ \\
\hline Asian & 0.851 & 1.085 & $0.463-2.541$ \\
\hline American Indian or Alaska Native & 0.04 & 2.118 & $1.033-4.343$ \\
\hline $\begin{array}{l}\text { Native Hawaiian or other Pacific } \\
\text { Islanders }\end{array}$ & 0.465 & 1.403 & $0.565-3.487$ \\
\hline \multicolumn{4}{|l|}{ Reoperation } \\
\hline Black or African American & 0.023 & 0.831 & $0.709-0.974$ \\
\hline Asian & 0.928 & 1.042 & $0.424-2.56$ \\
\hline American Indian or Alaska Native & 0.512 & 0.703 & $0.246-2.014$ \\
\hline $\begin{array}{l}\text { Native Hawaiian or other Pacific } \\
\text { Islanders }\end{array}$ & 0.449 & 1.455 & $0.551-3.842$ \\
\hline
\end{tabular}

and reported significantly longer length of stay and higher readmission rates in Black patients with both procedures. However, reoperation and re-intervention were significantly higher only in Black patients who underwent SG. They additionally showed significantly higher mortality rate in Black patients who had SG. Although race and specifically nonHispanic Black race has been reported as an independent predictor of mortality [19], the mortality rate in this study was $0.1 \%$ and we did not find any significant difference in this variable among racial groups in our study population. Additionally, we also observed significantly longer length of stay in African Americans in this study (Table 4), a disparity that has been reported in other fields of surgery as well, and one that Wahl et al. [20] successfully eliminated in a study between Black and White patients undergoing colorectal surgery with enhanced recovery after surgery protocols.

Tiwari and colleagues [21] have also used a database with more than 37,000 patients who underwent laparoscopic gastric bypass over 4 years and reported race as a risk factor affecting postoperative outcomes in these patients. They also reported that a 30-day readmission was significantly higher in non-Hispanic Blacks compared with non-Hispanic Whites. African American race has been reported as an independent risk factor for readmission in many other studies as well [22-24]. In this study, the majority of readmissions (78\%) were associated with the metabolic and bariatric procedure. Despite significant differences in cause of readmission among racial groups, nausea and vomiting, fluid, electrolyte, or nutritional depletion was the most common cause in all groups and the reason for almost one-fourth of all interventions after the primary procedure.

Postoperative complications were not listed separately in the multivariate logistic regression for each outcome model in order to reduce the number of variables and increase the accuracy of the analysis. However, we found results in univariate analyses that are comparable with previous studies. For instance, occurrence of PE was 1.93 times higher in AAs compared with Whites in our study, which may be secondary to a biological predilection, although this is unknown, and is especially puzzling since there were no significant differences in the preoperative rates of venous thromboembolism. This is comparable with the results of a study by Turner and colleagues [25] using the National Surgical Quality Improvement Program (NSQIP) database from 2005 to 2007 and reported increased rates of certain postoperative complications including 2.5 times higher PE in African American patients. Venous thrombosis was the third most common reason for readmission in AA patients in this study. Notably, preoperative IVC filter was significantly higher in AAs compared with White patients, while the preoperative rates of 
DVT or PE were not different between these two racial groups. However, over $80 \%$ of AAs who had IVC filter preoperatively received it in anticipation of the bariatric procedure as opposed to a pre-existing filter (18\%). These findings are not easily explained. The higher mean preoperative BMI in the AA group and possibly anticipated longer operation times could perhaps account for the significantly higher rates of preoperative IVC filter placement in AAs compared with Whites even though the preoperative PE and DVT rates were similar between the groups.

In addition to postoperative outcomes, there were some differences in the patient characteristics, comorbidities, and perioperative factors (Tables 1, 2, and 3) among different races that were adjusted for in the multivariate logistic regression. For instance, despite LSG being the most common procedure performed in all racial groups, LRYGB was performed with even lower rates in AAs compared with Whites, which is similar to what was reported by Worni and colleagues [26] on the trends of LRYGB among different races from 2002 to 2008. However, this difference in the two procedures declined to the point that they were performed in almost equal proportions in Native Hawaiian or other Pacific Islander patients.

We have also demonstrated significant variation in obesityrelated comorbidities among racial groups in this study population. American Indian or Alaska Native patients had the highest rate of diabetes (35.8\%), while African Americans had the highest rates of hypertension $(53.6 \%)$. These findings are comparable with those from the National Diabetes Statistics Report [27] and National Center for Health Statistics (NCHS) data brief on hypertension [28] by CDC in 2017, respectively.

Despite being the largest bariatric-specific dataset in the country with over 95\% 30-day patient follow-up rate, MBSAQIP database is associated with a number of limitations. First, we were only able to test the variables that were presented in the database. For instance, factors that have been shown to affect patient outcomes such as socio-economic and insurance status, center volume, and surgeon experience are not included in the database [19, 29, 30]. Second, the retrospective nature of the study precludes randomization of study groups; as a result, many of the variables differed significantly among racial groups and adjusting was required in the final analyses.

\section{Conclusion}

Postoperative complications, readmission, re-intervention, and reoperation are generally uncommon events within 30 days of bariatric surgery with $6,3.8,1.3$, and $1.2 \%$ prevalence respectively in those who underwent LRYGB or LSG captured in 2016 MBSAQIP PUF. However, these events occur disproportionately among different races. This study is one of the first to include other racial groups besides African American and White and report racial disparity in short-term postoperative outcomes of metabolic and bariatric procedures using 2016 MBSAQIP database. Knowing which racial groups are at risk for poorer outcomes can guide perioperative measures aimed at risk reduction. Lastly, the findings presented herein should provoke future studies on the etiology of these disparities as well as strategies by which they can be meaningfully addressed.

\section{Compliance with Ethical Standards}

Conflict of Interest The authors declare that they have no conflict of interest.

Ethical Approval This study has received exempt IRB approval from Rush University Medical Center IRB. For this type of study, formal consent is not required.

\section{References}

1. Centers for Disease Control and Prevention. National center for chronic disease prevention and health promotion, division of nutrition, physical activity, and obesity. Data, Trend and Maps [online];201903/07.

2. MacLean LD, Rhode BM, Nohr CW. Late outcome of isolated gastric bypass. Ann Surg. 231(4)

3. O'Brien PE, Hindle A, Brennan L, et al. Long-term outcomes after bariatric surgery: a systematic review and meta-analysis of weight loss at 10 or more years for all bariatric procedures and a singlecentre review of 20-year outcomes after adjustable gastric banding. Obes Surg. 29(1)

4. Pories WJ, Swanson MS, MacDonald KG, et al. Who would have thought it? An operation proves to be the most effective therapy for adult-onset diabetes mellitus. Ann Surg. 222(3)

5. Schauer PR, Ikramuddin S, Gourash W, et al. Outcomes after laparoscopic Roux-en-Y gastric bypass for morbid obesity. Ann Surg. 232(4)

6. Sjostrom L, Lindroos AK, Peltonen M, et al. Lifestyle, diabetes, and cardiovascular risk factors 10 years after bariatric surgery. $\mathrm{N}$ Engl J Med. 351(26)

7. Chen Y, Corsino L, Shantavasinkul PC, et al. Gastric bypass surgery leads to long-term remission or improvement of type 2 diabetes and significant decrease of microvascular and macrovascular complications. Ann Surg. 263(6)

8. Omotosho P, Mor A, Shantavasinkul PC, et al. Gastric bypass significantly improves quality of life in morbidly obese patients with type 2 diabetes. Surg Endosc. 30(7)

9. Turrell G, Vandevijvere S. Socio-economic inequalities in diet and body weight: evidence, causes and intervention options. Public Health Nutr. 18(5)

10. Parikh M, Hetherington J, Sheth S, et al. Frequencies of obesity susceptibility alleles among ethnically and racially diverse bariatric patient populations. Surg Obes Relat Dis. 9(3)

11. Buffington CK, Marema RT. Ethnic differences in obesity and surgical weight loss between African-American and Caucasian females. Obes Surg. 16(2)

12. Fuchs HF, Broderick RC, Harnsberger CR, et al. Benefits of bariatric surgery do not reach obese men. J Laparoendosc Adv Surg Tech A. 25(3) 
13. Bhogal SK, Reddigan JI, Rotstein OD, et al. Inequity to the utilization of bariatric surgery: a systematic review and meta-analysis. Obes Surg. 25(5)

14. Lutfi R, Torquati A, Sekhar N, et al. Predictors of success after laparoscopic gastric bypass: a multivariate analysis of socioeconomic factors. Surg Endosc. 20(6)

15. Anderson WA, Greene GW, Forse RA, et al. Weight loss and health outcomes in African Americans and whites after gastric bypass surgery. Obesity (Silver Spring). 15(6)

16. Omotosho PA, Rodriguez JA, Jain-Spangler K, et al. Predictors of long-term success after laparoscopic Roux-en-Y gastric bypass in African-American women. Surg Obes Relat Dis. 12(2)

17. MBSAQIP Participant use data file;2018March/22.

18. Sheka AC, Kizy S, Wirth K, et al. Racial disparities in perioperative outcomes after bariatric surgery. Surg Obes Relat Dis.

19. Nguyen GC, Patel AM. Racial disparities in mortality in patients undergoing bariatric surgery in the U.S.A. Obes Surg. 23(10)

20. Wahl TS, Goss LE, Morris MS, et al. Enhanced recovery after surgery (ERAS) eliminates racial disparities in postoperative length of stay after colorectal surgery. Ann Surg. 268(6)

21. Tiwari MM, Goede MR, Reynoso JF, et al. Differences in outcomes of laparoscopic gastric bypass. Surg Obes Relat Dis. 7(3)

22. Sun SX, Hollenbeak CS, Rogers AM. Readmissions following gastric bypass surgery. Obes Surg. 26(2)

23. Abraham A, Ikramuddin S, Jahansouz C, et al. Trends in bariatric surgery: procedure selection, revisional surgeries, and readmissions. Obes Surg. 26(7)
24. Garg T, Rosas U, Rivas H, et al. National prevalence, causes, and risk factors for bariatric surgery readmissions. Am J Surg. 212(1)

25. Turner PL, Oyetunji TA, Gantt G, et al. Demographically associated variations in outcomes after bariatric surgery. Am J Surg. 201(4)

26. Worni M, Guller U, Maciejewski ML, et al. Racial differences among patients undergoing laparoscopic gastric bypass surgery: a population-based trend analysis from 2002 to 2008. Obes Surg. 23(2)

27. Centers for Disease Control and Prevention. National Diabetes Statistics Report, 2017. Atlanta, GA: Centers for Disease Control and Prevention, U.S. Dept. of Health and Human Services.

28. Fryar CD, Ostchega Y, Hales CM, Zhang G, Kruszon-Moran D. Hypertension prevalence and control among adults: United States, 2015-2016. NCHS Data Brief. (289)289.

29. Dallal RM, Bailey L, Guenther L, et al. Comparative analysis of short-term outcomes after bariatric surgery between two disparate populations. Surg Obes Relat Dis. 4(2)

30. Flum DR, Salem L, Elrod JA, et al. Early mortality among Medicare beneficiaries undergoing bariatric surgical procedures. JAMA. 294(15)

Publisher's Note Springer Nature remains neutral with regard to jurisdictional claims in published maps and institutional affiliations. 